\title{
Editorial
}

Nephrology

\section{Glomerular Structure in Diabetes - Can It Predict the Future?}

\author{
Kathryn White \\ Newcastle University - EM Research Services, Faculty of Medical Sciences, Newcastle upon Tyne, UK
}

Researchers have been performing in-depth quantitative analysis of glomerular structural parameters for several decades [1] and have long ago established the link between structural abnormalities and renal function [2]. However, it is still unclear whether changes in glomerular structure are a cause or effect of diabetic nephropathy (DN). It is certainly likely that even if initially the lesions are a result of the diabetic milieu, they are themselves going to contribute to the progression.

The study of glomerular structure using both light and electron microscopy can provide insight into the natural history of DN and can also be used to assess the effectiveness of treatment with, for instance, renin-angiotensin system blocking agents. It has been shown that the diabetic lesions can be reversed after 10 years in type 1 patients receiving pancreas transplants [3]. But will detailed quantification of structural parameters predict which patients are likely to progress from normoalbuminuria (NA) to microalbuminuria (MA) and on to proteinuria $(\mathrm{P})$ and end-stage renal disease (ESRD)?

In order to predict progression, a study must, of course, be longitudinal. Many of the reported studies using biopsy data are cross-sectional, but there have been some longitudinal studies providing information on disease progression. For patients at later stages of $\mathrm{DN}$, studies have shown that glomerular podocyte parameters at baseline correlate with function at follow-up [4-6]. However, it could be argued that identifying progressors at this stage is too late as the lesions are advanced and cannot be reversed without pancreas transplantation. Similarly, it has been argued that the widely used clinical predictor of progression - MA - is imprecise and occurs when damage has already been done. If it was possible to identify patients who were likely to progress much earlier even at the NA stage - then it could be possible to target such patients with the aim of preventing the development of advanced lesions and maintaining glomerular function. The largest longitudinal study of NA type 1 patients is from a group in Minneapolis [7]; this study has been exploring the natural history of DN in these patients and analysing several structural parameters in order to determine which parameters identify those patients who are most likely to progress to P or ESRD.

The first structural change seen in the glomeruli of diabetic patients is the thickening of the glomerular basement membrane (GBM) [1], and increased GBM width has been shown to predict progression of DN in NA type 1 diabetic patients [7]. Interestingly, the increased GBM width predicts progression not only from NA to MA but also from NA to $\mathrm{P}$ and ESRD so is a strong independent predictor of DN risk.

In recent years, interest in glomerular structure has moved from the GBM and mesangium to the podocyte. In $\mathrm{DN}$ there is podocyte foot process widening or 'effacement' with loss of filtration slits. Foot process effacement should be reversible as long as the podocytes themselves

\section{KARGER 125}

(c) 2015 S. Karger AG, Base

$0250-8095 / 15 / 0415-0275 \$ 39.50 / 0$
Kathryn White

Newcastle University - EM Research Services

Faculty of Medical Sciences

Newcastle upon Tyne NE2 4HH (UK)

www.karger.com/ajn
E-Mail kathryn.white@ncl.ac.uk 
remain intact. However, extensive podocyte damage will eventually lead to podocyte loss. There is a great deal of literature on the proteins involved in regulating the function of the podocyte slit diaphragm and how disruption of this intricate structure results in $\mathrm{P}$ (reviewed in [8]). So could changes in podocyte structure in NA diabetic patients predict progression to later stages of disease? Harindhanavudhi et al. [9] have looked at podocyte structural parameters but have not found any differences in these parameters between progressors and non-progressors. This negative finding could be due to lack of precision in the estimate - parameters such as podocyte number, foot process width and filtration slit length tend to have a high degree of variability. Alternatively, it could suggest that ultrastructural changes to the podocyte occur later in the disease process. There is lack of agreement as to when podocytes are lost, particularly in type 1 diabetes. The current study does not show any reduction in podocyte number in NA type 1 patients, which is in contrast to an earlier study by Steffes et al. [10] but more consistent with a study in normotensive type 1 patients where podocyte number correlated inversely with AER in proteinuric but not microalbuminuric patients [6]. In the Pima Indians, increases in albuminuria combined with hypertension result in a dramatic loss of podocytes [5]. This suggests that ultrastructural changes to the podocyte occur later in the disease process and are due to the combined effect of both mechanical and metabolic stresses applied to the cell.

Diabetic patients are not routinely biopsied and so clinical biopsies may not be representative of the diabetic population. Therefore, it has been important to have research studies of diabetic patients, which have provided valuable insight into the pathology of the disease and how structure and function are related. However, longitudinal biopsy studies from diabetic patients are likely to become scarce as first of all there is an inherent risk in performing the biopsy, making ethical approval difficult to obtain. Second, the vast majority of patients are on anti-hypertensive treatment, which could influence the results and getting ethical approval to stop anti-hypertensive treatment will again be unlikely.

So if GBM width does predict progression from NA to $\mathrm{P}$ and ESRD is it, or any other structural parameter, likely to be taken up in the clinical setting? There is disagreement between clinicians and pathologists as to the necessity of performing biopsies on diabetic patients without other indications. The time-consuming method of GBM measurement using electron microscopy is also a deterrent. Perhaps we need to follow what we have learned from structural studies - that the GBM increases early in diabetes and GBM width can predict progression - and look for noninvasive ways to detect this structural change. Although the podocyte has become the focus of much diabetic (and other renal disease) research, perhaps it is time to go back to the less glamourous basement membrane. Are there noninvasive biomarkers that indicate changes to the composition of the GBM?

In conclusion, the usefulness of glomerular structural parameters as predictors of DN progression in the clinical setting is limited due to being invasive and time-consuming. However, detailed quantitative analysis of glomerular ultrastructure has contributed widely to our understanding of the pathology of $\mathrm{DN}$ and although it might not be able to tell us the future, it may have pointed us in the right direction.

\section{References}

1 Osterby R: Morphometric studies of the peripheral glomerular basement membrane in early juvenile diabetes. I. Development of initial basement membrane thickening. Diabetologia 1972;8:84-92.

2 Mauer SM, Steffes MW, Ellis EN, Sutherland DE, Brown DM, Goetz FC: Structural-functional relationships in diabetic nephropathy. J Clin Invest 1984;74:1143-1155.

3 Fioretto P, Steffes MW, Sutherland DE, Goetz FC, Mauer M: Reversal of lesions of diabetic nephropathy after pancreas transplantation. N Engl J Med 1998;339:69-75.

4 Meyer TW, Bennett PH, Nelson RG: Podocyte number predicts long-term urinary albu- min excretion in Pima Indians with type II diabetes and microalbuminuria. Diabetologia 1999;42:1341-1344.

5 Lemley KV, Abdullah I, Myers BD, Meyer TW, Blouch K, Smith WE, Bennett PH, Nelson RG: Evolution of incipient nephropathy in type 2 diabetes mellitus. Kidney Int 2000; 58:1228-1237.

6 White KE, Bilous RW, Marshall SM, El Nahas M, Remuzzi G, Piras G, De Cosmo S, Viberti G: Podocyte number in normotensive type 1 diabetic patients with albuminuria. Diabetes 2002;51:3083-3089.

7 Caramori ML, Parks A, Mauer M: Renal lesions predict progression of diabetic ne- phropathy in type 1 diabetes. J Am Soc Nephrol 2013;24:1175-1181.

8 Grahammer F, Schell C, Huber TB: The podocyte slit diaphragm - from a thin grey line to a complex signalling hub. Nat Rev Nephrol 2013;9:587-598.

9 Harindhanavudhi T, Parks A, Mauer M, Caramori ML: Podocyte structural parameters do not predict progression to diabetic nephropathy in normoalbuminuric type 1 diabetic patients. Am J Nephrol 2015;41:277-283.

10 Steffes MW, Schmidt D, McCrery R, Basgen $\mathrm{JM}$ : Glomerular cell number in normal subjects and in type 1 diabetic patients. Kidney Int 2001;59:2104-2113. 